\title{
Clinical Characterisation of Dystonia in Adult Patients with Huntington's Disease
}

NA van de Zande ${ }^{1,2}$, TH Massey (MD, PhD $)^{1,3}$, D McLauchlan $(M D)^{1}$, A Pryce Roberts $(M D)^{3}, R$ Zutt $(M D)^{4}, M$ Wardle $(M D)^{5,7}$, GC Payne $(M D)^{5}, C$ Clenaghan ${ }^{1}, M A J$ Tijssen (MD, PhD $)^{4}, A E$ Rosser $(\mathrm{MD}, \mathrm{PhD})^{1,6,7}, \mathrm{KJ}$ Peall $(\mathrm{MD}, \mathrm{PhD})^{1,3,7}$

${ }^{1}$ Huntington's Disease Research Group, Institute of Psychological Medicine and Clinical Neurosciences, Cardiff, UK.

${ }^{2}$ Faculty of Medical Science, University of Groningen, Groningen, The Netherlands.

${ }^{3}$ MRC Centre for Neuropsychiatric Genetics and Genomics, Hadyn Ellis Building, Cardiff, UK.

${ }^{4}$ Department of Neurology, University Medical Centre of Groningen, The Netherlands.

${ }^{5}$ Cardiff and Vale University Health Board, University Hospital of Wales, UK

${ }^{6}$ Cardiff University Brain repair Group, School Biosciences, UK

${ }^{7}$ Wales Brain Repair and Intracranial Neurotherapeutics Unit (BRAIN), Cardiff University, UK Corresponding Author

Dr Kathryn Peall, MRC Centre for Neuropsychiatric Genetics and Genomics, Hadyn Ellis Building, Cardiff, CF24 4HQ. Email: PeallKJ@cardiff.ac.uk, Telephone: 02920688353

Total Word count: 3470

Running Title: Characterisation of Dystonia in Huntington's Disease

Key Words: Huntington's Disease, Dystonia, Movement Disorders, Scales, Quality of Life All authors report no competing interests. 


\section{ABSTRACT}

\section{Background}

Huntington's disease (HD) is an autosomal dominant, neurodegenerative movement disorder, typically characterized by chorea. Dystonia is also recognised as part of the HD motor phenotype, although little work detailing its prevalence, distribution, severity and impact on functional capacity has been published to date.

\section{Methods}

Patients ( $>18$ years) were recruited from the Cardiff (United Kingdom) HD-clinic, each undergoing a standardised videotaped clinical examination and series of functional assessment questionnaires (Unified Huntington's Disease Rating Scale (UHDRS), Burke-FahnMarsden Dystonia Rating Scale (BFMDRS) and modified version of the Toronto Western Spasmodic Torticollis Rating Scale. The presence and severity of dystonia was scored by four independent neurologists using the BFMDRS and the UHDRS rating scales. Statistical analysis included Fisher's exact test, Wilcoxon test, ANOVA and calculation of correlation coefficients where appropriate.

\section{Results}

Forty-eight (91\% (48/53) demonstrated evidence of dystonia, with the highest prevalence in the left upper limb ( $n=44,83 \%)$, right upper limb most severely affected, and eyes least affected. Statistically significant positive correlations $(p<0.05)$ were observed between dystonia severity and increasing HD-disease stage and motor disease duration. Deterioration in functional capacity also correlated with increasing dystonia severity. No significant relationship was observed with age at motor symptom onset or CAG-repeat length. 


\section{Conclusions}

We report a high prevalence of dystonia in adult HD patients, with worsening dystonia severity with increasing HD-disease stage and motor disease duration. The recognition and management of dystonic symptoms in routine clinical practice will aid overall symptomatic treatment, and aid functional improvement. 


\section{INTRODUCTION}

Huntington's disease (HD) is an autosomal dominant, neurodegenerative disorder characterized by disorders of movement, cognition and behaviour, and caused by a trinucleotide (CAG) repeat expansion in the huntingtin (HTT) gene.[1] Chorea is the most widely recognised type of movement disorder associated with HD, although dystonia, tremor, myoclonus, ataxia and tics are also recognised.[2-4] Dystonia involves loss of coordinated contraction of antagonistic muscle groups, altering muscle tone and leading to abnormal movement and postures.[5] Few previous studies have sought to characterise dystonia in patients with $\mathrm{HD}$, with those that have tending to involve clinical descriptions of smaller cohorts.[6] Several groups have observed that dystonia appears most prominent in those with juvenile HD (onset $<20$ years) and longer CAG repeat length, while others have noted a positive correlation between dystonia severity and motor disease duration. $[7,8]$ In spite of this, dystonia remains difficult to assess in the presence of chorea, and tends to be overlooked in HD in favour of treating the chorea.

Dystonia is widely considered to be a neuronal circuit disorder involving the basal ganglia, striatum, cerebellum, and motor cortices.[9] The exact nature of this disruption remains poorly understood but is likely to involve altered monoaminergic neurotransmission, impacting functional connectivity between selected brain regions. Striatal atrophy is a prominent feature of HD pathogenesis with recent work identifying preferential loss of parvalbuminergic (PARV+) interneurons contributing to the dystonia phenotype.[10] An improved characterisation of dystonia in HD may aid in improving our understanding of the mechanisms that give rise to distinct clinical phenotypes. 
This study involves the largest cohort of genetically confirmed HD patients to undergo systematic and standardised assessment of the rate, distribution and severity of dystonia. We have sought to identify potential clinical markers associated with the presence and severity of dystonia, whether CAG repeat length correlates with dystonic symptoms, and how dystonia may impact on an individual's functional capacity.

\section{METHODS}

Participants $>18$ years of age with a confirmed pathogenic CAG expansion ( $>39$ repeats) in the HTT gene, were invited to participate in the study, via the South Wales HD Service (MayOctober 2016). All participants provided informed consent or third party assent where appropriate ('Move Wales: Welsh Movement Disorders Research Network REC Ref: 14/WA/0017).

\section{Clinical Data Collection}

Baseline clinical data collection included HD disease stage, age at motor symptom onset, past and present medication and CAG repeat length. HD disease stage was determined by the physician at their most recent clinical consultation, principally determined by functional ability.[11] Participants underwent a standardised videotaped clinical examination using a modified form of the Burke-Fahn-Marsden Dystonia Rating Scale protocol (BFMDRS), a series of functional capacity questionnaires including the Unified Huntington's Disease Rating Scale (UHDRS), BFMDRS functional capacity questionnaire and a modified version of the Toronto Western Spasmodic Torticollis Rating Scale (Disability Section) (TWSTRS).[12-14] A question from the Abnormal Involuntary Movement Scale (AIMS) was used to determine participant 
awareness of dystonia.[15] Distribution of chorea was determined from that observed during the videotaped clinical examination.

\section{Assessment of Videotaped Clinical Examination}

The videotaped examinations were reviewed by four independent neurologists, each with expertise in movement disorders and use of the UHDRS and BFMDRS rating scales, and blinded to all clinical information other than the genetic diagnosis of HD. Each reviewer was given detailed written instructions of how to apply the rating scales, and an opportunity to review the standardised UHDRS and BFMDRS videotaped clinical examinations. Each videotaped examination was scored using the UHDRS dystonia section (maximum score 20) and BFMDRS (maximum score 120). The UHDRS Dystonia scale is subdivided into five body regions (trunk, right and left upper and lower limbs) each scored between 0 and 4, while the BFMDRS scores severity and provoking factors in nine body regions (eyes, face, speech and swallow, neck, trunk, right and left upper and lower limbs) each being score between 0 and 16. The sum of scores for each rating scale was calculated per neurologist, and the mean of this value used for further analysis. Dystonia was considered to be present in an individual region when three or more of the scoring neurologists noted its presence.

\section{Statistical Analysis}

Statistical analysis involved use of the R statistical software package with Fisher's exact test, ANOVA, Pearson's correlation coefficients, normal regression and Wilcoxon signed rank test calculations used where appropriate. The interclass correlation coefficient (ICC) was used to determine the inter-rater reliability between clinicians and the intra-reliability of two raters re-scoring 10 of the original videos after an interval of 8 weeks. A p-value $<0.05$ was 
considered to be significant.

\section{RESULTS}

Fifty-three symptomatic and pre-manifest patients (32M: 21F), with genetically confirmed HD were recruited. Median age at examination was 49 years (range: $24-78$ years) with a mean duration of motor symptoms of 5.93 years. Eight patients (15.1\%) were receiving treatment with neuroleptic medication at the time of assessment, with two further individuals having taken this form of medication in the past. There was no reported temporal relationship between the onset of dystonia and use of neuroleptic medication, and no clinical evidence of tardive dyskinesia. Information relating to HTT CAG repeat length was available in 50 participants with a mean length of 43 repeats (standard deviation: 2.73 ). Genetic testing in the remaining three was performed elsewhere and although confirmed to involve a pathogenic CAG expansion, the exact number of repeats was unknown. A summary of the principal demographic characteristics can be seen in Table 1.

Dystonia was identified in at least one body region in $91 \%$ of patients $(48 / 53)$. Of the five individuals considered not to have any evidence of dystonia, three or more of the raters agreed that there was evidence of dystonia but not to its distribution. Excluding these five patients, the most commonly affected body regions were the Left $(44 / 48,83 \%)$ and Right (41/48, 77\%) Upper Limbs. Overall mean dystonia severity score was 32.18 (standard deviation: 28.21) using the BFMDRS, and 7.57 (standard deviation: 4.92) using the UHDRS. Dystonia severity was most marked in the Upper Limbs using the BFMDRS (mean scores: Right (5.42), Left (5.39)), and in the trunk (including the cranio-facial region) (mean score: 1.76) using the UHDRS. Using the recently updated dystonia classification system, a 
generalized dystonia distribution was most commonly observed $(n=32)$, followed by multifocal ( $n=10)$, segmental $(n=4)$ and focal $(n=2)$ forms. [16]

Evaluation of the inter-rater reliability scores was considered excellent (inter-rater reliability ICC of 0.91 (UHDRS) and 0.95 (BFMDRS)). Test-Rest evaluation determined ICC values of 0.81 and 0.96 (UHDRS) and 0.94 and 0.98 (BFMDRS) (Supplementary Table 1). The mean values and variance for each rater can be seen in Supplementary Figures 2 and 3. Agreement between three of the four raters was allowed as confirmation of the presence of dystonia in each case, although agreement between all four raters was seen in the majority of cases $(47 / 53(89 \%))$.

Evaluation of dystonia severity with respect to HD disease stage demonstrated a gradual increase in mean dystonia severity scores from premanifest to late stages (Table 1 and Figure 1). Further analysis found a significant increase in dystonia severity between each stage using both standardised tools, BFMDRS ( $p$-value: $<2 e-16$ ) and UHDRS ( $p$-value: $<2 e-$ 16). A statistically significant positive correlation was also observed between dystonia severity and motor disease duration (UHDRS (Correlation Coefficient: 0.82, Cl: 0.70-0.89, pvalue: 9.73e-14), BFMDRS (Correlation Coefficient: 0.84, Cl: 0.74-0.90, p-value: 4.23e-15) (Figure 2), and dystonia severity and disease burden score (UHDRS (Correlation Coefficient: $0.62 \mathrm{Cl}$ : (0.42-0.77), p-value: 1.18e-06), BFMDRS (Correlation Coefficient: $0.57 \mathrm{Cl}$ : (0.350.73), p-value: 1.34e-05) (Table 2). No significant relationship was seen between dystonia severity, CAG repeat length and age at motor symptom onset (Table 2 and Figure 2). 
Fifty-one participants completed the functional capacity questionnaires (UHDRS, BFMDRS and modified TWSTRS) to determine the impact of the movement disorder on day-to-day living. These demonstrated a significant correlation $(p<0.05)$ between dystonia severity and functional capacity, such that more impaired daily functioning was observed with increasing dystonia severity (Table 2 and Supplementary Figure 1). We also noted a significant correlation between increasing chorea severity and worsening functional capacity, although this effect appeared smaller than that observed with dystonia (BFMDRS $(p=0.008)$, UHDFCS $(p=0.0007)$, aTWSTRS $(p=0.0002)$. Only 17 patients $(33 \%)$ were aware of their dystonic movements, with 7 (14\%) individuals reporting some degree of distress, ranging between mild $(3(6 \%))$, moderate $(3(6 \%))$ and severe $(1(2 \%))$.

\section{DISCUSSION}

This study is the first to use systematic and standardised assessment tools to determine the presence, severity and distribution of dystonia in a large cohort of patients with genetically confirmed HD. Evidence of dystonia was documented in $91 \%$ of this cohort, with dystonia severity positively correlated with duration of motor symptoms at time of assessment, disease burden score and increasing stage of HD. The upper limbs were the most commonly and severely affected body part, with reduced functional capacity with worsening dystonic symptoms.

Previous studies have described rates of up to $95.2 \%$ of dystonia in HD cohorts, reflecting the strong prevalence of dystonia as part of the HD motor phenotypic spectrum, although there are other large cohort studies with no description of dystonia.[17] Louis et al examined 42 patients describing internal rotation of the shoulder (64.3\%), sustained fist clenching 
(47.1\%) and foot inversion (42.9\%). However, they reported only four cases of

blepharospasm (9.5\%) and no evidence of facial or laryngeal dystonia, compared to the 28 (53\%), 35(66\%) and 36(68\%) respectively seen in this study.[7] A possible explanation for this lies in the standardised scales used to assess this cohort. We sought to use a dystoniaspecific tool (BFMDRS) to provide the level of detail used to assess primary dystonic disorders. Here the scale grades the severity and provoking factors in contrast to the binary scale used by Louis et al, hence providing greater scope in diagnosis, although has likely led to an over estimation, particularly of laryngeal and ocular involvement. Previous reports have also described bruxism in patients with HD however, there was no evidence of teeth grinding during the examination, and no reported dental problems in this cohort.[18]

Previous studies have provided some conflict in those factors most likely to impact the presence and severity of dystonia. In this study we found HD disease stage, motor disease duration and disease burden score to be related to the severity of dystonic symptoms. Stage of HD disease is predominantly determined by degree of impairment in day-to-day living, with previous studies noting the same increase in dystonia severity with disease stage as was observed in this cohort. $[7,19]$ This idea is further supported by results from the multiple functional capacity assessments undertaken in this study (UHDRS, BFMDRS and adapted TWSTRS). We found increasing dystonia severity to be overwhelmingly associated with worsening functional capacity using all three scales, suggesting that a comprehensive assessment of dystonic symptoms is essential in the symptomatic management of patients with HD. However, it should also be noted that these patients were also of later disease stage and longer motor disease duration, and therefore other motor and non-motor factors are also likely to impact their overall functional capacity. 
A further conflict of findings is seen with age at motor symptom onset and CAG repeat length in relation to dystonia. This contrasts with findings from previous studies that have suggested that dystonia, or 'atypical movements' are more frequently observed in HD patients with a younger age at onset. $[2,8,20]$ However, both of these latter studies focused on patients with atypical patterns of presentation, potentially suggesting that the findings from this cohort are more representative of the wider spectrum of motor phenotype. These findings are also important in the context of Juvenile HD, cases of which were not included in this study. Here the motor phenotype is considered to be more bradykinetic with more pronounced dystonia.[21] However, given the specific pattern of motor symptomatology it may be applicable to consider those with onset of symptoms $<20$ years as a distinct subgroup.

Treatment of movement disorders is often complicated in disorders such as HD where different forms may co-exist in the same body part. Treatment of dystonia is dependent on its distribution, with botulinum toxin injections used for focal or segmental muscle involvement, and anti-cholinergic medical therapy (e.g. trihexyphenidyl) and tetrabenazine for more generalised symptoms.[5, 22] Of the participants recruited in this study only one was receiving ongoing botulinum toxin treatment for cervical dystonia, reporting moderate symptomatic improvement. Alternative treatment options include Deep Brain Stimulation (DBS), although a recent prospective pilot study $(n=6)$ found pallidal stimulation to improve dystonic symptoms in only $50 \%$ of the cases examined.[23] The potential benefits of cannabinoids in HD have also been explored, with the American Academy of Neurology guidelines suggesting that although anecdotal evidence indicates improvement to non- 
chorea symptoms in HD, randomised controlled studies have failed to demonstrate a significant symptomatic improvement.[24]

Symptomatic treatment strategies are typically determined by the spectrum of patient reported symptoms. Interestingly, in both this and previous studies, patients have reported little awareness of their dystonic symptoms. The patients in this study were able to describe a clear distinction between their dystonic and choreic symptoms with none having marked cognitive impairment at the time of assessment. Although, more advanced disease and cognitive impairment are likely to impact this recognition and subsequent reporting.[7] However, treatment strategies also seek to target functional improvement. Given the functional disability noted with increased dystonia severity in this study, an attempt at treating dystonic symptoms may result in improved functional capacity and overall daily living.

This study is principally limited by its cross-sectional design, providing only a single assessment and not reflecting daily and diurnal variation. We should also not exclude the possibility that the dystonia observed may be caused by factors other than HD. However, past or present use of neuroleptic agents was recorded in under a fifth of cases (10 (18.9\%)), and although a concurrent primary focal dystonia remains possible, it would be unlikely to explain the high frequency of dystonia observed. Finally, frequent use of the BFMDRS scale in the routine clinical setting is unlikely given the time required to complete alongside other routine clinical measures. The UHDRS dystonia scale performed to a similar high standard, although it should be noted that face, neck and trunk are included in a single score, and 
separating these into trunk and cranio-cervical components may provide a more robust measure, particularly in the guidance of therapeutic management.

\section{CONCLUSIONS}

This study represents the largest single cohort of patients with genetically confirmed HD to undergo systematic assessment for dystonia using standardised scales. We have demonstrated a significant association of increased dystonia severity with increasing HD stage, disease burden score and longer motor disease duration. In addition, functional capacity in day-to-day living worsened with increasing dystonia severity, reflecting the importance of its inclusion in ongoing management strategies. Further studies will be needed to confirm these findings in a larger cohort, however, development of the UHDRS Dystonia rating scale to distinguish cranio-cervical and truncal regions may aid in including this as part of routine clinical data collection.

\section{ACKNOWLEDGEMENTS}

We would like to thank all of the patients from the HD-clinic in Cardiff who have underwent the videotaped examinations and questionnaires.

\section{FUNDING}

KJP is a WCAT fellow, funded by an Academy of Medical Sciences Clinical Starter Grant and Life Sciences Research Network Wales Post-Doctoral Grant. THM is a WCAT and MRC Clinical Research Training Fellow (MR/P001629/1). MAJT is funded by Fonds Nuts-Ohra, Prinses Beatrix Fonds, Gossweiler Foundation, Fonds Psychische gezondheid, Phelps Stichting, Hersenstichting, Stichting Beatrix kinderziekenhuis, Stichting Wetenschapsfonds Dystonie 
Vereniging, the Parkinson patienten vereniging and unrestricted educational grants from Ipsen, Allergan, Merz, Acthelion and Medtronic. In January 2016 she received honorarium for a dystonia course in Paris by Merz. AER is funded by the Medical Research Council and a European Union FP7 Grant.

\section{COMPETING INTERESTS}

All authors report no competing interests. Disclosures: none.

\section{CONTRIBUTORS}

NvdZ, AER and KJP were involved in the design and conceptualisation of the study, analysis and interpretation of data, and drafting and revising of the manuscript for intellectual content. THM, DM, APR and RZ were involved in the assessment of the videotaped examinations and drafting and revising of the manuscript for intellectual content. MW and GP were involved in the analysis and interpretation of data, and drafting and revising of the manuscript for intellectual content. MAJT and CC were involved in the drafting and revising of the manuscript for intellectual content. 


\section{FIGURES}

Figure 1: Relationship between HD disease stage and dystonia severity

Legend: Figure of all scores, showing an increasing severity with a more advanced staged.

BFMDRS: Burke Fahn Marsden Dystonia Rating Scale, HD: Huntington's Disease, UHDRS:

Unified Huntington's Disease Rating Scale.

Figure 2: Relationship between dystonia severity and motor disease duration

Legend: Scatterplot with regression line showing an increase in severity with an increase of motor disease duration. BFMDRS: Burke Fahn Marsden Dystonia Rating Scale, UHDRS:

Unified Huntington's Disease Rating Scale.

\section{SUPPLEMENTARY TABLES}

Supplementary Table 1: Inter- and Intra-Rater Reliability Scores per Rater

Legend: BFMDRS: Burke Fahn Marsden Dystonia Rating Scale, $\mathrm{Cl}$ : Confidence Interval, ICC: Intra-class Correlation Coefficient, UHDRS: Unified Huntington's Disease Rating Scale

\section{SUPPLEMENTARY FIGURES}

Supplementary Figure 1: Relationship between dystonia severity and functional capacity Legend: Scatterplot showing decreased functional capacity with an increased severity of dystonia. BFMDFRS: Burke Fahn Marsden Dystonia Functionality Rating Scale, UHDFCS: Unified Huntington's Disease Functional Capacity Scale.

Supplementary Figure 2: Mean and variance of UHDRS Dystonia severity scores per rater 
Legend: Central line indicates the mean score per rater, and boxplot whiskers denote the variance in scores.

Supplementary Figure 3: Mean and variance of BFMDRS Dystonia severity scores per rater Legend: Central line indicates the mean score per rater, and boxplot whiskers denote the variance in scores. 


\section{REFERENCES}

1. Richards RI. Dynamic mutations: a decade of unstable expanded repeats in human genetic disease. Hum Mol Genet. 2001;10(20):2187-94.

2. Squitieri F, Berardelli A, Nargi E, Castellotti B, Mariotti C, Cannella M, et al. Atypical movement disorders in the early stages of Huntington's disease: clinical and genetic analysis. Clin Genet. 2000;58(1):50-6.

3. Becker N, Munhoz RP, Raskin S, Werneck LC, Teive HA. Non-choreic movement disorders as initial manifestations of Huntington's disease. Arq Neuropsiquiatr. 2007;65(2B):402-5.

4. Jankovic J, Ashizawa T. Tourettism associated with Huntington's disease. Mov Disord. 1995;10(1):103-5.

5. Albanese A, Asmus F, Bhatia KP, Elia AE, Elibol B, Filippini G, et al. EFNS guidelines on diagnosis and treatment of primary dystonias. Eur J Neurol. 2011;18(1):5-18.

6. Ashizawa T JJ. Cervical dystonia as the initial presentation of Huntington's disease. Mov Disord. 1996;11(4):457-9.

7. Louis ED, Lee P, Quinn L, Marder K. Dystonia in Huntington's disease: prevalence and clinical characteristics. Mov Disord. 1999;14(1):95-101.

8. Mahant N, McCusker EA, Byth K, Graham S, Huntington Study G. Huntington's disease: clinical correlates of disability and progression. Neurology. 2003;61(8):1085-92.

9. Niethammer M, Carbon M, Argyelan M, Eidelberg D. Hereditary dystonia as a neurodevelopmental circuit disorder: Evidence from neuroimaging. Neurobiol Dis. 2011;42(2):202-9. 10. Reiner A, Shelby E, Wang H, Demarch Z, Deng Y, Guley NH, et al. Striatal parvalbuminergic neurons are lost in Huntington's disease: implications for dystonia. Mov Disord. 2013;28(12):1691-9.

11. Shoulson I. Huntington disease: functional capacities in patients treated with neuroleptic and antidepressant drugs. Neurology. 1981;31(10):1333-5.

12. Burke RE, Fahn S, Marsden CD, Bressman SB, Moskowitz C, Friedman J. Validity and reliability of a rating scale for the primary torsion dystonias. Neurology. 1985;35(1):73-7.

13. Unified Huntington's Disease Rating Scale: reliability and consistency. Huntington Study Group. Mov Disord. 1996;11(2):136-42.

14. Consky ES, Lang AE. Clinical assessments of patients with cervical dystonia. In: Jankovic J, Hallett M, editors. Therapy with botulinum toxin. New York: Marcel Dekker; 1994. p. 211-37.

15. Abnormal Involuntary Movement Scale (AIMS). Psychopharmacol Bull. 1988;24(4):781-3.

16. Albanese A, Bhatia K, Bressman SB, Delong MR, Fahn S, Fung VS, et al. Phenomenology and classification of dystonia: a consensus update. Mov Disord. 2013;28(7):863-73.

17. Foroud T, Gray J, Ivashina J, Conneally PM. Differences in duration of Huntington's disease based on age at onset. J Neurol Neurosurg Psychiatry. 1999;66(1):52-6.

18. Tan EK, Jankovic J, Ondo W. Bruxism in Huntington's disease. Mov Disord. 2000;15(1):171-3.

19. Feigin A, Kieburtz K, Bordwell K, Como P, Steinberg K, Sotack J, et al. Functional decline in Huntington's disease. Mov Disord. 1995;10(2):211-4.

20. Louis ED, Anderson KE, Moskowitz C, Thorne DZ, Marder K. Dystonia-predominant adultonset Huntington disease: association between motor phenotype and age of onset in adults. Arch Neurol. 2000;57(9):1326-30.

21. Roos RA. Huntington's disease: a clinical review. Orphanet J Rare Dis. 2010;5:40.

22. Cloud LJ, Jinnah HA. Treatment strategies for dystonia. Expert Opin Pharmacother. 2010;11(1):5-15.

23. Wojtecki L, Groiss SJ, Ferrea S, Elben S, Hartmann CJ, Dunnett SB, et al. A Prospective Pilot Trial for Pallidal Deep Brain Stimulation in Huntington's Disease. Front Neurol. 2015;6:177.

24. Koppel BS, Brust JC, Fife T, Bronstein J, Youssof S, Gronseth G, et al. Systematic review: efficacy and safety of medical marijuana in selected neurologic disorders: report of the Guideline Development Subcommittee of the American Academy of Neurology. Neurology. 2014;82(17):155663. 
Characterisation of Dystonia in Huntington's Disease van de Zande et al 